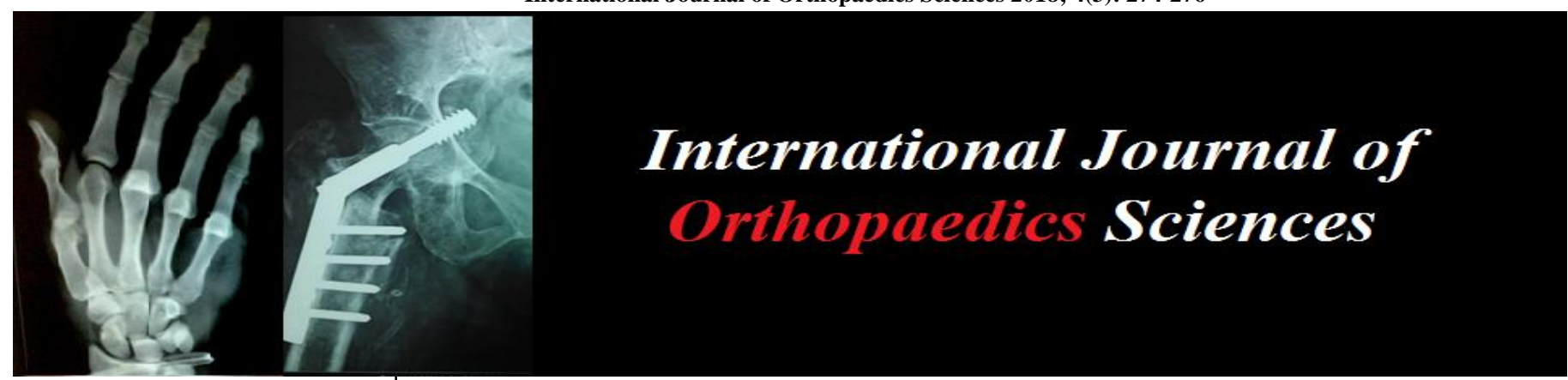

ISSN: $2395-1958$

IJOS 2018; 4(3): 274-276

(C) 2018 IJOS

www.orthopaper.com

Received: 18-05-2018

Accepted: 19-06-2018

Dr. Gokul Anand Murugesan

Mahatma Gandhi Medical

College and Research Institute

Pondy - Cuddalore Main Road,

Pondicherry, India

Dr. Dhiyanesh Krishnamurthy

Mahatma Gandhi Medical

College and Research Institute

Pondy - Cuddalore Main Road,

Pondicherry, India
Correspondence

Dr. Dhiyanesh Krishnamurthy Mahatma Gandhi Medical

College and Research Institute

Pondy - Cuddalore Main Road,

Pondicherry, India

\section{Comparative study between dynamic hip screw Vs proximal femoral nail for trochanteric fracture of femur}

\section{Dr. Gokul Anand Murugesan and Dr. Dhiyanesh Krishnamurthy}

DOI: $\underline{\text { https://doi.org/10.22271/ortho.2018.v4.i3e.48 }}$

Abstract

Trochanteric fractures are among the most common fractures encountered by an orthopaedic surgeon. These fractures were commonly encountered in the elderly population. Proximal femoral nail (PFN) and Dynamic hip screw (DHS) being the most commonly used. There is increased in usage of nails in the management of this fracture. Both the implants have own complication. We included 50 consecutive patients with tronchandic fracture who underwent PFN/DHS were included in this study. We analyzed the for pain over the operated site, range of motion and Harris hip score, radiological assessment for the screw position and neck shaft angle and union of fracture were assessed in this both groups. Based on our study, though the blood loss, shortening, neck shaft angle, had significant difference in early follow up, The functional result remained the same both in DHS and PFN at 1 year follow up. Hence PFN/DHS are safe implant of choice in the management of this fracture.

Keywords: Intertrochantric fracture, proximal femoral nail, dynamic hip screw

\section{Introduction}

Trochanteric fractures of femur are among the most common fractures encountered by an orthopaedic surgeon accounting for a good percentage of all fractures admitted. Over the past fifty years, a wide variety of implants and fixation strategies have been utilized for the surgical stabilization of intertrochanteric fractures.

The sliding compression hip screw and side plate became the standard of care for the surgical treatment of these fractures. To overcome implant related complications and facilitate the operative treatment of unstable peritrochanteric fractures, several intramedullary fixation devices of different design were introduced ${ }^{[1]}$.

PFN and DHS being the most commonly used and widely available, we have used the same implants and did this study. The average time, duration until union, functional outcome of intertrochanteric fractures operated with DHS/PFN were assessed in this study.

\section{Materials and methods}

This is a prospective study conducted in Mahatma Gandhi medical college and research institute, India, from august 2007 to July 2009. Randomization of fifty patients into two groups was done. Group A was treated with dynamic hip screw fixation and group B with proximal femoral nail fixation. In this study we used Boyd and Griffin classification for classifying the fracture pattern. All patients' sustained intertrochanteric fracture were included. The patients with associated fracture neck of femur, shaft of femur, pathological fractures, preexisting femoral deformity and previous surgery on the ipsilateral hip or femur were excluded from the study. Preoperatively, the patient's age, sex, mode of injury, mobility and hemoglobin were recorded. Approval for this prospective study was obtained from our local institutional ethical committee board.

In all fifty patients only spinal anesthesia was used and positioned with a fracture table. Fracture reduction under fluoroscopic guidance in fracture table done by closed reduction technique. If a closed reduction cannot be achieved after one or two attempts with percutaneous techniques, limited open reduction was used. Then the fixation of fracture was 
done using dynamic hip screw or proximal femoral nail according to randomization method in routine manner.

Follow up at 1, 3, 6, 9, and 12 months was done. At each visit patients were clinically assessed for pain over the operated site, range of motion and Harris hip score, radiological assessment for the screw position and neck shaft angle and union of fracture were noted. Statistical Comparison was done using Chi-square test.

\section{Results}

In this study, out of fifty patients, the youngest patient age is 19 and oldest patient is 90 years. The mean age in the DHS group was 61.26 years and PFN group was 57.68 years. Out of 50 patients, 33 were male and 17 were female. A 3:2 male to female ratio was noted. 28 patients had right sided intertrochanteric fracture and 22 had left sided fracture. On the basis of Boyd and Griffin classification, type I was 6, type II was 27, type III was 9 and type IV was 8 . Stable fracture in DHS group was 15 and PFN group were 17. Unstable fracture in DHS group was 8 and PFN group were 10.
Approximately around $72 \%$ patient had significant comorbidity, mainly diabetes mellitus, hypertension, ischemic heart disease. The mean timing of operation after fracture is 8.92 days in DHS group and 6.68 days in PFN group. The length of incision and blood loss in PFN group average $3.3 \mathrm{~cm}$ and $196 \mathrm{ml}$ respectively, in DHS group $7.8 \mathrm{~cm}$ and $315 \mathrm{ml}$.

No immediate and late complication in DHS group but in PFN, there was one screw back out due to the wrong entry point, which was taken from the lateral cortex of the femur. There was no wound infection and nonunion of fracture. $48 \%$ of patient treated with DHS returned to same job in PFN 52\% returned to same job.

\section{Statistical analysis}

We used Chisquare test to calculate the $\mathrm{p}$ value. The average duration of surgical procedure in DHS group was $68 \mathrm{~min}$ whereas in the PFN group it was 90min. This difference was significant. $(\mathrm{p}=0.02)$. The preop and post-operative mobility score in the DHS group 5.1, 4.6 and in the PFN group score was $6.3,5.7$. This difference was not significant.

\begin{tabular}{|c|c|c|c|}
\hline Variable & Dhs & Pfn & P value \\
\hline Mean operative time (in min) & $68+\_0.25$ & $90+\_0.19$ & $0.0282^{*}$ \\
\hline Blood loss in ml & $315+\_31.12$ & $196+\_26.4$ & $0.00^{*}$ \\
\hline Mean shortening & $1.2+\_0.24$ & $0.87+\_0.20$ & $0.00^{*}$ \\
\hline Mean neck shaft angle & $123.96+\_1.014$ & $129.60+\_1.685$ & $0.00^{*}$ \\
\hline
\end{tabular}

The mean intra operative blood loss was $315 \mathrm{ml}$ in the DHS group and in the PFN group was $196 \mathrm{ml}$. The difference was significant. $(p=0.00)$. Despite the difference in peroperative blood loss, the hemoglobin levels and the requirements for blood transfusion were similar in both groups. The fluoroscopy time in the DHS group was 2.02 and in the PFN group was 2.84. This difference was not significant.The shortening in the DHS group $1.2 \mathrm{~cm}$ and in PFN group was $0.87 \mathrm{~cm}$. This difference was significant $(\mathrm{p}=0.00)$. The mean neck shaft angle in DHS group is $\mathbf{1 2 3 . 9 6}$ and in PFN group is 129.6 degree. This difference also was significant $(p=0.00)$. There was no significant difference in the hospital stay of those who were discharged between the two groups. Although the total mobility score was similar in the two treatment groups at six and twelve months, the ability to walk outside was significantly better at those time periods for the patients who had an intramedullary hip screw. The use of assistive devices at twelve months was not found to differ between the two treatment groups. The mean time to full weight bearing was 1.3 months in DHS group and in the PFN group it was around 1.2 months. This was not significant.

In the DHS group excellent result was $15(60 \%)$, good was 7 $(28 \%)$, fair was $2(8 \%)$ and poor $1(4 \%)$ and in the PFN group excellent was $17(68 \%)$, good was $5(20 \%)$, fair was $1(4 \%)$ and poor was $2(8 \%)$. Harris hip function score was more or less same in one year follow up. In DHS group, the score was 84 and in PFN group it was 86. Comparing both the result of DHS and PFN group, the p value is 0.9908 which is greater than 0.05 , hence statistical significance was unable to achieve.

\section{Discussion}

A wide range of proximal femoral fracture fixation devices have been employed over the years, the sliding compression hip screw and side plate (DHS) became the implant of choice for the fixation of intertrochanteric fractures in the latter half of the twentieth century ${ }^{[2]}$. Several intramedullary devices came to exist in order to overcome the shortcomings of the extramedullary implant. In this study, we had compared the sliding compression hip screw and side plate (DHS) and the proximal femoral nail in the fixation of intertrochanteric femoral fractures.

D.C.R. Hardy et al. ${ }^{[3]}$, in a prospective study of 100 patients were treated with PFN and DHS for intertrochanteric femoral fractures noted the mean pre op mobility score was $4.4+2.9$ and the post op score was 3.4+_3.41, in this study the pre op score was $5.1+2.76$, and the post op score was $4.6+2.84$ in DHS group. In the PFN group the mean pre op mobility score was $5.2+\_3.3$ and the post op score was $5.3+$ 3.03, in this study the pre op score was $6.3+3.2$, and the post op score was $5.7+2.4$. Early rehabilitation was found in the nail group was due to the fact that sliding of the lag screw does not occur in the nail, mostly act as a static device, which accounts for the less shortening, but it does not affect the functional outcome of the patient.

Pajarinen ${ }^{[4]}$ et al. also noted similar findings that PFN group patients were more likely to have regained their pre op level of mobility than those in the DHS group. They suggested that the impaction of the fracture in the DHS group led to femoral neck shortening and a mechanical disadvantage compared with the proximal femoral nail.

In this study also shows that the time taken to mobilise with a frame is shorter with the PFN than with the DHS. The reason for this may be any combination of postoperative factors: pain, muscle dysfunction and small incision above the greater trochanter. This entry point causes less damage to the superior gluteal nerve and gluteus medius muscle than other entry points in the piriformis fossa ${ }^{[5]}$. The DHS, however, requires a larger incision and probably damages more muscle.

The per operative blood loss was significantly less with the PFN probably because of closed operative technique which requires only a $3 \mathrm{~cm}$ incision and a small split in the abductor muscle. By contrast the DHS needs a much longer incision and elevation of vastus lateralis. Also we performed only minimum reaming of the femoral shaft to reduce medullary blood loss. Despite these advantages we were unable to show any benefit for the patients, since the post-operative 
hemoglobin levels and transfusion requirements of the two groups were similar, perhaps because most blood loss occurs at the fracture itself before and after surgery.

We found less sliding of the lag screw after the intramedullary hip screw procedures as has been noted by other investigators ${ }^{[6]}$. The intramedullary nail stops the telescoping displacement of the proximal aspect of the femur. In fact, the proximal part of the nail blocks the head and neck fragment, preventing its complete impaction. Thus, there is less subsequent shortening of the affected limb.

Some authors have suggested that intramedullary hip screw fixation is superior for certain subsets of patients, particularly those with fractures that are "unstable", such as fractures with reverse obliquity patterns, fractures with lateral wall or posteromedial comminution, and fractures extending into the femoral neck or subtrochanteric regions. These types are not well controlled by the sliding compression hip screw and side plate, and they are associated with a high rate of fixation failure when treated with that type of implant ${ }^{[7]}$.

Complication in the nail was shaft fracture due to inappropriate reaming one case occurred in Hardy study ${ }^{[3]}$ but no cases in this study. Next concern is the mid thigh pain which occurred in the nail group due to distal locking screw, in this study we had three patient with persistent pain which resolved later in the follow up visit. Thus we strongly recommend that only in the unstable pattern, usage of two distal locking screws is beneficial.

Hardy et al. ${ }^{[3]}$ noted that the result were quite similar in both the group. However because of the overall shortening of the limb, the possibility of early weight bearing, better early walking score suggest that the intramedullary device is a promising alternative only in comminuted intertrochanteric fracture and those with the reverse oblique fracture pattern. In this study also on comparing both the result of DHS and PFN group found no statistical significant difference.

\section{Conclusion}

From our study, we found PFN had significant benefits over DHS in terms of blood loss, shortening, neck shaft angle, which has significant $\mathrm{p}$ value. But in the functional result wise both the DHS and PFN remains the same.

\section{References}

1. Simmermacher RK, Bosch AM, Van der Werken C. The AO/ASIF - proximal femoral nail (PFN): Anew device for the treatment of unstable proximal femoral fractures. Injury. 1999; 30:327-332.

2. Babst R, Renner N, Biedermann M, Rosso R, Heberer M, Harder F, Regazzoni P. Clinical results using the trochanter stabilizing plate (TSP): the modular extension of the dynamic hip screw (DHS) for internal fixation of selected unstable intertrochanteric fractures. J Orthop Trauma. 1998; 12:392-399.

3. Hardy DC, Descamps PY, Krallis P, Fabeck L, Smets P, Bertens CL et al. Use of an intramedullary hip-screw compared with a compression hip-screw with a plate fprintertrochanteric femoral fractures. A prospective, randomized study of one hundred patients. J Bone Joint Surg Am. 1998; 80:618-630.

4. Pajarinen J. Pertrochanteric femoral fracture treated with dynamic hip screw or a proximal femoral nail. JBJS. 2005; 87-B:76-81.

5. Moein CM, Verhofstad MH, Bleys RL, Van der Werken C. Soft tissue injury related to choice of entry point in antegrade femoral nailing: piriformis fossa or greater trochanter tip. Injury. 2005; 36:1337-42.

6. Bridle SH, Patel AD, Bircher M, Calvert PT. Fixation of intertrochanteric fractures of the femur. A randomized prospective comparison of the gamma nail and the dynamic hip screw. J Bone Joint Surg. 1991; 73B(2):330-334.

7. $\mathrm{Wu} \mathrm{CC}$, Shih $\mathrm{CH}$. Biomechanical analysis of the dynamic hip screw in the treatment of intertrochanteric fractures. Arch Orthop and Trauma Surg. 1991; 110:307310. 